EPJ Web of Conferences 81, 04002 (2014)

DOI: $10.1051 /$ epjconf/ 20148104002

(C) Owned by the authors, published by EDP Sciences, 2014

\title{
Quarkonium production and polarization in pp collisions with the CMS detector
}

\author{
Alessandro Degano $^{1, a}$ for the CMS Collaboration \\ ${ }^{1}$ University of Turin, INFN and CERN
}

\begin{abstract}
Studies of the production of heavy quarkonium states are very important to improve our understanding of QCD and hadron formation, given that the heavy quark masses allow the application of theoretical tools less sensitive to nonperturbative effects. Thanks to a dedicated dimuon trigger strategy, combined with the record-level energy and luminosity provided by the LHC, the CMS experiment could collect large samples of pp collisions at 7 and $8 \mathrm{TeV}$, including quarkonium states decaying in the dimuon channel. This allowed the CMS collaboration to perform a series of systematic measurements in quarkonium production physics, including double-differential cross sections and polarizations, as a function of rapidity and $p_{T}$, for five $\mathrm{S}$-wave quarkonia: $\mathrm{J} / \psi, \psi(2 \mathrm{~S}), \Upsilon(1 \mathrm{~S})$, $\Upsilon(2 S)$, and $\Upsilon(3 S)$. Some of these measurements extend well above $p_{T} \simeq 100 \mathrm{GeV}$, probing regions of very high $p_{T}$ /mass, where the theory calculations are supposed to be the most reliable. Thanks to its high-granularity silicon tracker, CMS can reconstruct lowenergy photons through their conversions to $\mathrm{e}^{+} \mathrm{e}^{-}$pairs, thereby accessing the radiative decays of the P-wave quarkonium states, with an extremely good mass resolution, so that the $\mathrm{J}=1$ and $\mathrm{J}=21 \mathrm{P}$ states can be resolved. This allows CMS to determine cross-section ratios and feed-down decay fractions involving the $\chi$ states, in both the charmonium and bottomonium families.

This talk presents some of the most recent CMS quarkonium production results, in pp collisions, in particular the production of $\Upsilon(1 \mathrm{~S}, 2 \mathrm{~S}, 3 \mathrm{~S})$, the cross-section ratio of charmonium and bottomonium P-wave states and the polarization of S-wave $c \bar{c}$ and $b \bar{b}$ states.
\end{abstract}

\section{Introduction}

Around the early 90's theorists thought that quarkonium production in hadron collisions would be relatively simple to describe using the Color Singlet Model (CSM). In this model, $q \bar{q}$ mesons form as a consequence of the hard scattering of the quark and anti-quark in a color singlet state. The situation became interesting when CDF measured a $J / \psi$ and $\psi(2 S)$ production cross section that exceeded CSM predictions by almost a factor 50 [1]. While for the $J / \psi$ the discrepancy could be ascribed to the incomplete knowledge of the feed-down from P-wave states $\left(\chi_{c}\right)$, the tension of the $\psi(2 S)$ crosssection result between theory and experiment was a strong hint that the theoretical framework was incomplete. Non-relativistic QCD (NRQCD) was invented to solve this tension. NRQCD is an effective theory that features the factorization of perturbative short-distance processes, that describe the hard scattering, and non-perturbative long-distance matrix elements (LDMEs) that account for the

\footnotetext{
ae-mail: alessandro.degano@cern.ch
} 
formation of the meson. The LDMEs are process independent and must be extracted phenomenologically. NRQCD predicts transverse polarization for the S-wave states of quarkonium produced in hadron collisions. Pre-LHC polarization measurements were not able to confirm or disconfirm this prediction in a definite way [2].

The CMS experiment is giving a very important contribution to the field, measuring cross section and polarization of five S-wave quarkonium states with unprecedented precision and novel analysis techniques. Understanding P-wave quarkonia is very important to complete the picture, and the latest CMS results are presented in the following.

\section{Recent results from CMS: $\Upsilon$ production}

The CMS detector is described in detail in [3]. Although not specifically designed for quarkonium physics, it features a high-performance silicon tracker, a 3.8T solenoid and a muon system that make it very suitable for detecting decays of S-wave quarkonia into a pair of muons. The tracker is also used to detect low-energy photons emitted in radiative transitions of P-wave quarkonia by reconstructing conversions into an $e^{+} e^{-}$pair. The flexible trigger system also plays a key role to make the study of quarkonia possible in CMS. All the results presented here rely on di-muon triggers.

Several S-wave states cross-section measurements were published by the CMS collaboration [5, 6]. Here we start by describing the latest results on the measurement of the production cross-section of $\Upsilon(1 S), \Upsilon(2 S)$ and $\Upsilon(3 S)$ in pp collisions at $\sqrt{s}=7 \mathrm{TeV}$. The measurement presented here [8] is based on $4.9 \mathrm{fb}^{-1}$ of data taken in 2011 and extends the coverage in transverse momentum $\left(p_{T}\right)$ of the $\Upsilon$ up to $100 \mathrm{GeV}$. The rapidity region studied is restricted to $|y(\mu \mu)|<0.6$. The behavior of the differential cross sections for higher- $n \mathrm{~S}$-wave relative to lower- $n \mathrm{~S}$-wave states is studied. The technique consists in measuring the event yield $N_{\Upsilon(n S)}^{f i t}$ using an unbinned maximum likelihood fit to the data, and extracting the cross section times the branching ratio to $\mu^{+} \mu^{-}, \sigma(p p \rightarrow \Upsilon(n S)) \times$ $\mathcal{B}\left(\Upsilon(n S) \rightarrow \mu^{+} \mu^{-}\right)$, by applying a per-event muon efficiency correction $\epsilon_{\mu \mu}$ derived from data:

$$
\frac{d \sigma(p p \rightarrow \Upsilon(n S))}{d p_{T}} \times \mathcal{B}\left(\Upsilon(n S) \rightarrow \mu^{+} \mu^{-}\right)=\frac{N_{\Upsilon(n S)}^{f i t}\left(p_{T}\right)}{L_{\text {int }} \cdot \Delta p_{T} \cdot \epsilon_{\mu \mu}\left(p_{T}\right) \cdot \mathcal{A}\left(p_{T}\right)}
$$

with $\mathcal{A}$ the detector acceptance, measured with simulation taking into account the results of the CMS polarization measurement [9]. The results for the differential production cross sections of $\Upsilon(1 S)$, $\Upsilon(2 S)$ and $\Upsilon(3 S)$ are illustrated in figure 1 . The same figure also shows the behavior, as a function of $p_{T}$, of the ratio of the production cross sections of $\Upsilon(2 S)$ and $\Upsilon(1 S)$.

\section{P-wave quarkonia}

P-wave quarkonia are an essential piece of the puzzle of quarkonium production. Feed-down from $\mathrm{P}$-wave states must be taken into account when studying S-wave production and polarization. Relative production cross sections of P-waves are interesting tests of (NR)QCD per se. Moreover, measurements of production cross sections and polarizations of $\mathrm{P}$-wave states can help constraining the LDMEs.

The measurement of the ratio of the production cross sections of $\chi_{c 2}$ and $\chi_{c 1}$ is appealing because several theoretical uncertainties (e.g. quark masses or the value of $\alpha_{s}$ ) as well as experimental uncertainties (trigger efficiencies, integrated luminosity) cancel out. P-wave states are generally studied using their radiative decays to a ${ }^{3} S_{1}$ state plus photon. Excellent photon energy reconstruction is needed to separate the $\mathrm{J}=2$ from the $\mathrm{J}=1$ state. For this purpose CMS can exploit the potential of its 
MESON 2014 $-13^{\text {th }}$ International Workshop on Production, Properties and Interaction of Mesons
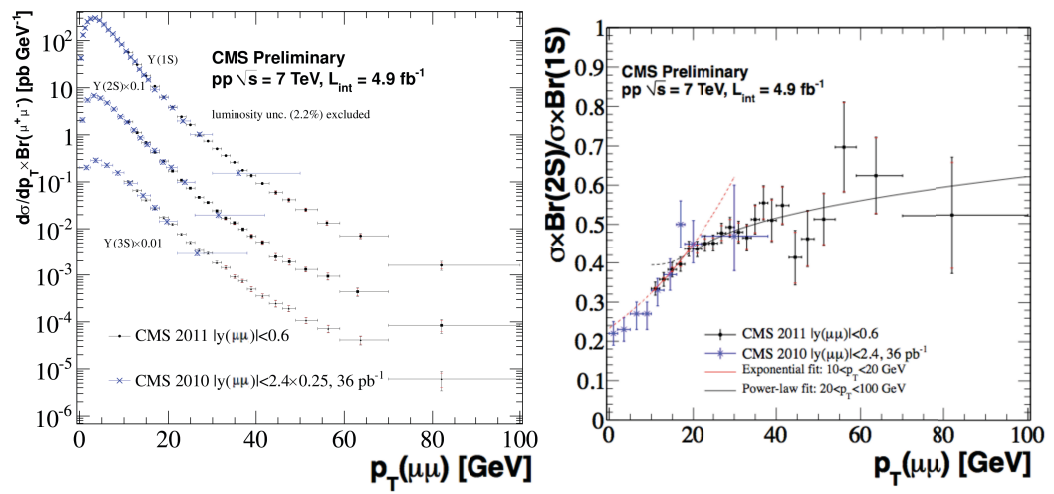

Figure 1. Left: Measurement of the production cross section of $\Upsilon(1 S), \Upsilon(2 S)$ and $\Upsilon(3 S)$. The $\Upsilon(2 S)$ and $\Upsilon(3 S)$ cross sections are scaled by 0.1 and 0.01 for clarity. Right: ratio of the production cross sections of $\Upsilon(2 S)$ and $\Upsilon(1 S)$

silicon tracker and its $3.8 \mathrm{~T}$ magnetic field. When photon energy is measured using conversions in the tracker, the resolution is around $5 \mathrm{MeV}$ for photons in the energy range of $\chi_{c}$ and $\chi_{b}(1 P)$ decays. The measurement of the ratio of the production cross sections of $\chi_{c 2}$ and $\chi_{c 1}$ with the CMS experiment is published in [10], where the results are compared to two theoretical predictions using the $k_{T}$ factorization and NRQCD. The former seems unable to reproduce the data, while the latter shows good agreement with the experiment. It must be however pointed out that NRQCD offers more degrees of freedom, since LDMEs are extracted from a fit to experimental data.

The same type of measurement, this time in the bottomonium sector, and recently published [11] is presented by CMS at this conference. The decays $\chi_{b 2,1}(1 P) \rightarrow \Upsilon(1 S)+\gamma$ are studied using the photon conversion technique. Figure 2 shows the invariant mass $m_{\mu \mu \gamma}$, extracted from a kinematic fit, in one of the $p_{T}$ ranges of the $\Upsilon$ under study. It also shows the behavior of the ratio of the cross sections in the four bins of $p_{T}$ of the $\Upsilon$ under study.
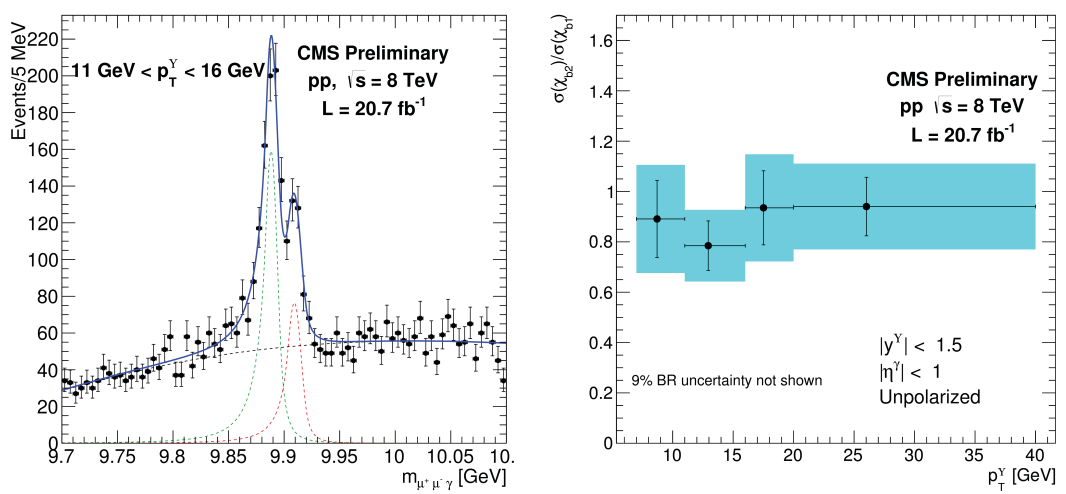

Figure 2. Left: invariant mass of the $\mu \mu \gamma$ system in the $\chi_{b}(1 P)$ region Right: ratio of the production cross sections of $\chi_{b 2}(1 P)$ and $\chi_{b 1}(1 P)$ presented by CMS. 


\section{$4 \mathrm{~J} / \psi, \psi(2 S)$ and $\Upsilon(1 S, 2 S, 3 S)$ polarization}

NRQCD predicts that the ${ }^{3} S_{1}$ states of quarkonium are produced transversely polarized at high $p_{T}$ in hadron collisions. Previous measurements were inconclusive with this respect [2]. CMS measured the polarization of all five ${ }^{3} S_{1}$ states in three different polarization frames in [12] and [9]. The data show no evidence of transverse polarization. In particular, the $\psi(2 S)$ and $\Upsilon(3 S)$ states are the ones for which the polarization can be interpreted unambiguously, since they are less affected by the feed-down of P-wave states.

The experimental results for the polarization parameter $\tilde{\lambda}$ of $J / \Psi$ and $\psi(2 S)$ are shown in figure 3 evaluated in different helicity frames. No evidence of transverse polarization is present as it would be characterized by $\tilde{\lambda} \simeq 1$.

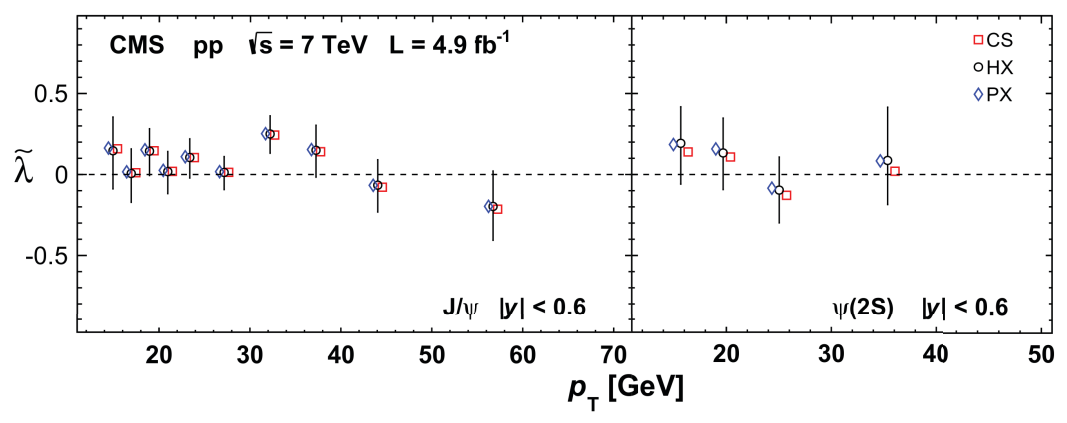

Figure 3. Polarization parameter $\tilde{\lambda}$ for $\mathrm{J} / \Psi$ and $\Psi(2 \mathrm{~S})$ : results exclude large transverse or longitudinal polarization

\section{Conclusions}

The problem of production and polarization of quarkonium in hadron collisions offers a unique insight for the understanding of strong interactions. While state-of-the-art theory (QCD) seems to be able to explain production cross-sections reasonably well, the absence of transverse polarization of ${ }^{3} S_{1}$ states remains a puzzle. The CMS experiment has presented several precision results in this field, which will provide useful experimental ground for a better grasp of hadron formation mechanisms.

\section{References}

[1] F. Abe et al. (CDF Collaboration), Phys. Rev. Lett. 79 (1997) 572

[2] P. Faccioli et al., Eur. Phys. J. C69 (2010) 657

[3] CMS Collaboration, JINST 3 (2008) S08004

[5] CMS Collaboration, JHEP 02 (2012) 011

[6] CMS Collaboration, Phys. Lett. B 727 (2013) 101

[8] CMS Collaboration, CMS-PAS-BPH-12-006, http://cds . cern. ch/record/1548008? ln=en

[9] CMS Collaboration, Phys. Rev. Lett. 110 (2013) 081802

[10] CMS Collaboration, Eur. Phys. J. C72 (2012) 2251

[11] CMS Collaboration, CMS-PAS-BPH-13-005, http://cds . cern. ch/record/1633613? ln=en

[12] CMS Collaboration, Phys.Lett. B727 (2013) 381-402 